\title{
The potential role of granulosa cells in the maturation rate of immature human oocytes and embryo development: A co-culture study
}

\author{
Bahia Namavar Jahromi' , Zahra Mosallanezhad ${ }^{2}$, Najmeh Matloob ${ }^{3}$, Maryam Davari ${ }^{3}$, Mohamed Amin Ghobadifar ${ }^{4}$ \\ 'Infertility Research Center, Department of Obstetrics and Gynecology, Division of Reproductive Endocrinology and Infertility, ${ }^{2}$ Student Research \\ Committee, Department of Obstetrics and Gynecology, School of Medicine, Shiraz University of Medical Sciences, Shiraz; ${ }^{3}$ nfertility Research Center, \\ Mother and Child Hospital, Shiraz; ${ }^{4}$ Zoonoses Research Center, Faculty of Medicine, Jahrom University of Medical Sciences, Jahrom, Iran
}

Objective: In order to increase the number of mature oocytes usable for intracytoplasmic sperm injection (ICSI), we aimed to investigate the effect of co-culturing granulosa cells (GCs) on human oocyte maturation in vitro, the fertilization rate, and embryo development.

Methods: A total of 133 immature oocytes were retrieved and were randomly divided into two groups; oocytes that were cultured with GCs (group A) and oocytes that were cultured without GCs (group B). After in vitro maturation, only oocytes that displayed metaphase II (MII) underwent the ICSI procedure. The maturation and fertilization rates were analyzed, as well as the frequency of embryo development.

Results: The mean age of the patients, their basal levels of follicle-stimulating hormone, and the number of oocytes recovered from the patients were all comparable between the two study groups. The number of oocytes that reached MII (mature oocytes) was 59 out of 70 (84.28\%) in group $A$, compared to 41 out of $63(65.07 \%)$ in group $B(p=0.011)$. No significant difference between fertilization rates was found between the two study groups $(p=0.702)$. The embryo development rate was higher in group $\mathrm{A}(33 / 59,75 \%)$ than in group $\mathrm{B}(12 / 41,42.85 \% ; p=0.006)$. The proportion of highest-quality embryos and the blastocyst formation rate were significantly lower in group $B$ than in group $A(p=0.003$ and $p<0.001$, respectively).

Conclusion: The findings of the current study demonstrate that culturing immature human oocytes with GCs prior to ICSI improves the maturation rate and the likelihood of embryo development.

Keywords: Fertilization; Granulosa cells; Intracytoplasmic sperm injection; In vitro oocyte maturation techniques; Oocytes

\section{Introduction}

Human oocytes remain in the dictyate stage after birth until gonadotropin stimulates them to reinitiate meiosis at the time of ovula-

Received: May 1, 2015 · Revised: Jul 9, 2015 · Accepted: Aug 25, 2015 Corresponding author: Mohamed Amin Ghobadifar

Zoonoses Research Center, Faculty of Medicine, Jahrom University of Medical Sciences, Motahari Avenue, Postal Code 193, Jahrom, Iran

Tel: +98-936-620-8078, +98-917-10-20-790 Fax:+98-713-635-40-94

E-mail:amin_m505@yahoo.com

*This article was partly based on the thesis written by Zahra Mosallanezhad, and was financially supported by the Shiraz University of Medical Sciences (grant number 92-01-50-6445).

This is an Open Access article distributed under the terms of the Creative Commons Attribution Non-Commercial License (http://creativecommons.org/licenses/by-nc/3.0/) which permits unrestricted non-commercial use, distribution, and reproduction in any medium, provided the original work is properly cited. tion. Until fertilization occurs, human oocytes enter metaphase II (MII). Immature human oocytes in pre-ovulatory non-atretic follicles can experience in vitro maturation (IVM) from germinal vesicle (GV) breakdown to the arrest of MII [1]. Pincus and Enzmann [2] first reported the IVM of mammalian immature oocytes. In vitro fertilization (IVF) and IVM of immature oocytes obtained from non-stimulated ovaries have been performed in farm animals, resulting in pregnancy and birth [3]. The use of immature human oocytes for the initiation of pregnancy was first documented in the studies of Cha et al. [4] and Trounson et al. [5]. Moreover, the IVM procedure has the advantages of having a lower cost and minimizing the risk of ovarian hyperstimulation syndrome, since it does not require the use of expensive gonadotropin [1].

A series of mutual interactions between human oocytes and their 
surrounding somatic cells are required for the development of functional ovarian follicles in vivo. It was once thought that oocytes are exclusively passive recipients of developmental signals from the somatic cells that they communicate with. However, data from studies performed over the past decade have revealed that the communication between oocytes and their surrounding somatic cells is bidirectional [6].

Gap junctions and paracrine signaling have been described as the two modes of intercellular connection between an oocyte and its companion somatic granulosa cells (GCs) [7]. Cell-to-cell gap junction communication connects oocytes to GCs, and GCs to other GCs, allowing these cell types to function together metabolically. In contrast, signaling molecules that are too large for transmission through gap junctions bind to target cells, followed by secretions into the extracellular space. Although paracrine communication and gap junctions are potentially bidirectional, GC-to-oocyte connections are mediated by paracrine signals, whereas the majority of oocyte-to-GC communication takes place through gap junctions [8].

The culture conditions used for the IVM of oocytes can significantly affect maturation rates and the likelihood of subsequent embryo development. A particularly crucial component for normal cytoplasmic oocyte maturation is the presence of GCs or cumulus cells surrounding the oocytes. Notably, oocyte metabolism, and in particular, how oocytes use energy sources, is regulated by GCs [9]. Moreover, it is known that GCs can protect the oocyte against oxidative stress, be- cause they undergo apoptosis during IVM [10]. During the performance of micromanipulation techniques, such as intracytoplasmic sperm injection (ICSI), the human oocyte is denuded (stripped of its surrounding G(s) and immature oocytes are often discarded. With this in mind, in order to increase the number of appropriate mature oocytes usable for ICSI procedures, we planned to investigate the effect of co-cultures of GCs on the IVM of human oocytes, their fertilization rate, and embryo development.

\section{Methods}

\section{Study population and oocyte recovery}

This cross-sectional prospective study was carried out among women undergoing IVF treatment using their own oocytes in the Department of Infertility and Reproductive Medicine at the Shiraz University of Medical Sciences between May 2013 and August 2014. Eighty young women were considered for eligibility, and 52 consenting women 20 to 35 years of age were ultimately enrolled into the study consecutively (Figure 1). From these women, 133 immature oocytes were retrieved and were randomly divided into oocytes cultured with GCs (group A) and oocytes cultured without GCs (group B). Immature oocytes were defined using a slightly modified version of the method described by Russell [11]. Basal follicle-stimulating hormone (FSH) levels $<10 \mathrm{mlU} / \mathrm{mL}$ and patients undergoing their first IVF treatment were the inclusion criteria. None of the women in the study groups

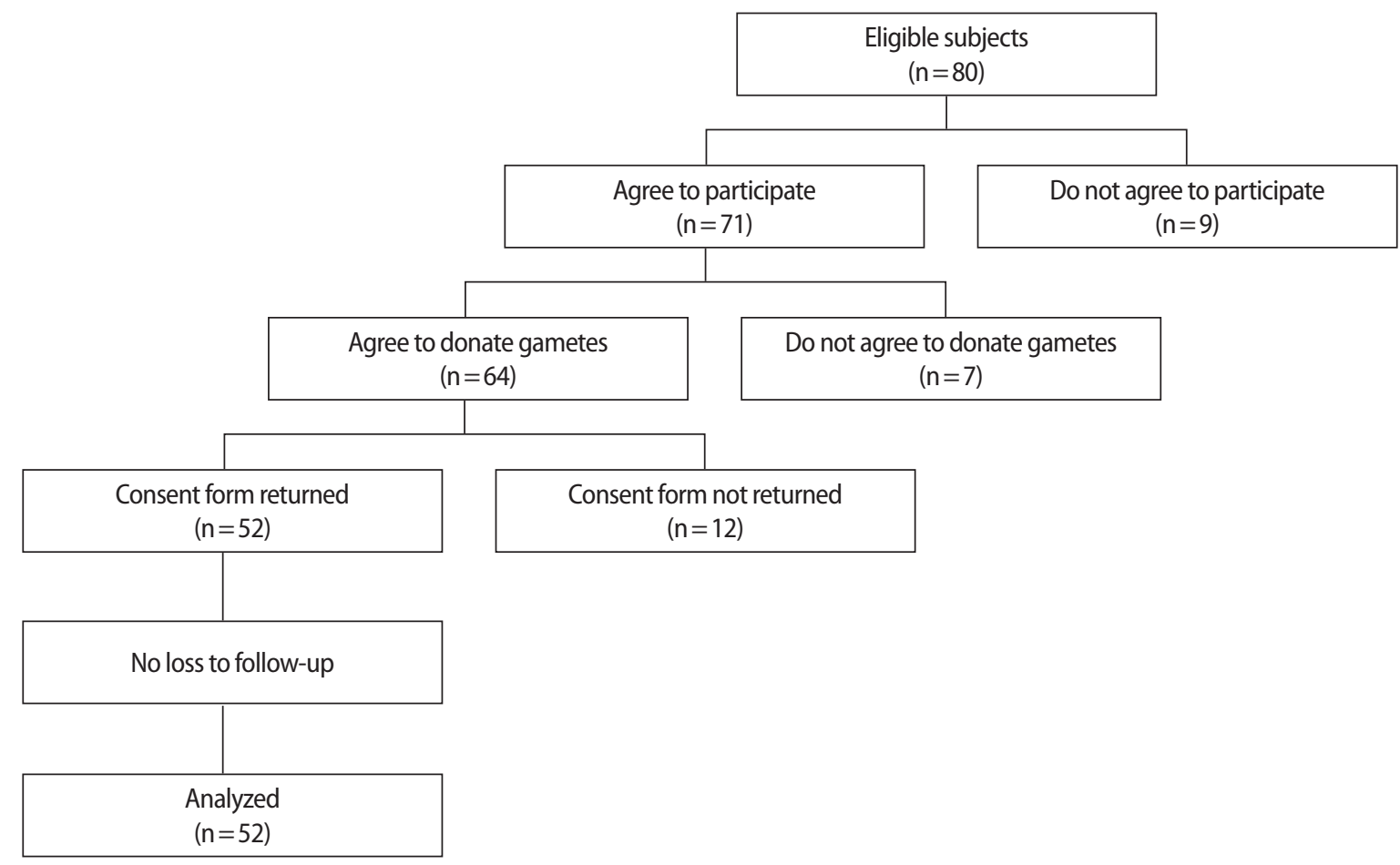

Figure 1. Flow chart of the requirements for participation in the study. 
had a history of systemic disease or polycystic ovary syndrome. At least one immature oocyte was retrieved in each cycle. The study was undertaken after being completely approved by the Institutional Review Board of the Shiraz University of Medical Sciences. Written informed consent was obtained from each infertile couple before the use of their gametes.

A flexible gonadotropin-releasing hormone $(\mathrm{GnRH})$ antagonist protocol was implemented [12]. For all patients, the starting dose of recombinant FSH (Menogon, Baxter Oncology, Mumbai, India) was 150 IU/day on the second day of the cycle. The daily administration of 0.25 mg of cetrorelix (Baxter Oncology) was initiated when the presence of at least one follicle measuring $>14 \mathrm{~mm}$ was noted. Treatment with the $\mathrm{GnRH}$ antagonist and recombinant FSH continued daily until the day when the final maturation of the oocytes was triggered. An injection of 10,000 IU of human chorionic gonadotropin (hCG) (Pregnyl, N.V.Organon, Oss, The Netherlands) was administered when half of the mature follicles were at least $17 \mathrm{~mm}$ in diameter, as measured in two planes. Transvaginal oocyte retrieval was scheduled for approximately 34 to 36 hours after hCG administration.

\section{In vitro maturation, fertilization, and embryo development}

Approximately 2 to 4 hours after retrieval, the majority of GCs were dissected from the granulosa-oocyte complexes. For denudation of the oocytes, $80 \mu \mathrm{L}$ drops of a $1 \%$ solution of hyaluronidase (Sigma, Cleveland, $\mathrm{OH}, \mathrm{USA}$ ) were used. The immature oocytes were then retrieved and were randomly divided into oocytes cultured with GCs (group A) and oocytes cultured without GCs (group B). The method of co-culturing GCs with immature oocytes has been previously described [13]. In both groups, IVM culture media (Sigma) was used 24 hours prior to ICSI. An incubator at $37^{\circ} \mathrm{C}$ with saturated humidity and an atmosphere of $5 \% \mathrm{CO}_{2}$ and $6 \% \mathrm{O}_{2}$ was used to culture the immature oocytes. After IVM, only oocytes that displayed a first polar body were classified as being in MII and underwent the ICSI procedure. The spermatozoa samples were dispersed by centrifugation with human tubal fluid (HTF) medium (Sigma) supplemented with 10\% fetal bovine serum.

Spermatozoa were injected based on the method described by Van Steirteghem et al. [14]. Subsequently, all injected oocytes were cultured in a drop of potassium simplex optimized medium (Sigma) and covered with oil. Each oocyte was examined for fertilization and structural integrity 18 hours after this process. Normal fertilization was confirmed by the observation of two polar bodies and two distinct pronuclei (PN) under an inverted microscope. The zygotes were then transferred into P-1 medium with $10 \%$ synthetic serum substitute (Sigma) and covered with mineral oil. Based on small modifications of the criteria described by Puissant et al. [15], the embryo quality was assessed 72 hours after the ICSI procedure. The extent of fragmenta- tion and the number of blastomeres were counted and recorded. Embryos were scored and classified based on the amount of detached anuclear fragments, the symmetry of the blastomeres, and the number of blastomeres. The amount of detached anuclear fragments was scored as follows: scores of $0,1,2,3$, and 4 were given to embryos in which detached anuclear fragments comprised $>50 \%, 26 \%$ to $50 \%$, $11 \%$ to $25 \%, 6 \%$ to $10 \%$, and $0 \%$ to $5 \%$ of the volume, respectively. The symmetry of the blastomeres was scored as follows: a score of 1 was given to embryos with symmetric blastomeres, and a score of 0 was given to embryos with asymmetric blastomeres. In order to score the number of blastomeres, a score of 1 was given to embryos with two to four cells, a score of 2 was given to embryos with five cells, a score of 3 was given to embryos with six or seven cells, and a score of 4 was given to embryos with eight to ten cells. The final score for each embryo was obtained by adding the scores for the three parameters described above. For instance, the final score of asix-cell (scored 3) embryo with asymmetrical blastomeres (scored 0) and 7\% fragmentation (scored 3) was 6 . Scores $<5$ were considered to indicate non-viable embryos, and scores $\geq 6$ were considered to indicate embryos appropriate for ICSI. Scores of 9 or 10 were interpreted as indicating highest-quality embryos [15].

\section{Statistical analysis}

Based a power of $90 \%$ ( $p=0.05$, two-sided) for finding a significant difference similar to the findings of the preliminary study [13], $96 \mathrm{im}$ mature oocytes were found to be a suitable sample size for the current study. In order to compensate for any refusal on the part of the subjects, we decided to collect 133 immature oocytes on the retrieval day. The results were reported as percentages for categorical variables, and mean \pm standard deviation or median for quantitative variables. The Student's $t$-test was applied to compare parametric data sets. The chi-squared test was used to compare fertilization rates, IVM, and embryo development. Two-sided $p$-values $<0.05$ were considered to indicate statistical significance. All statistical analyses were performed using SPSS ver. 17.0 (SPSS Inc., Chicago, IL, USA).

\section{Results}

All participants completed the study. The average age of the subjects was $22.58 \pm 1.96$ years. A combination of infertility risk factors was reported in $71 \%$ of the couples. Single risk factors for infertility were as follows: endometriosis (11\%), male factor infertility (8.5\%), tubal factor infertility (4.1\%), ovulatory dysfunction (2.7\%), and other risk factors (2.6\%). The mean age, basal levels of FSH, and the number of oocytes recovered from each patient were all comparable between the two study groups. Altogether, 70 and 63 immature oocytes were included in group $A$ and group $B$, respectively. In both study groups, all of the 
collected immature oocytes were at the GV stage. The number of oocytes that reached MII (mature oocytes) was 59 of $70(84.28 \%)$ in group $A$, compared to 41 of $63(65.07 \%)$ in group $B(p=0.011)$. Clinical data regarding the participants and the rates of oocyte maturation in both study groups are shown in Table 1.

No significant difference between fertilization rates was found between the two study groups ( $p=0.702)$. Of the 59 mature oocytes in group A, 49 exhibited PN formation (91.83\% of which were mature oocytes with two PN, while $8.17 \%$ had 1 PN or 3 PN). Of the 41 mature oocytes in group B, 33 exhibited PN (90.9\% of which were mature oocytes with 2 PN, while $9.1 \%$ had 1 PN or 3 PN). No significant difference regarding normal fertilization rates (oocytes with 2 PN) was observed between the study groups ( $p=0.879$ ) (Table 2).

No significant difference in the embryo cleavage rate was found between the two study groups ( $p=0.417$ ). The rate of viable embryos in group A was significantly higher than in group B (75\% vs. $42.8 \%$, $p=0.006)$. The frequency of highest-quality embryos and cleavage stage (> six cells) embryos in group A was lower than in group B. In group $A$, the number of embryos with $<10 \%$ fragmentation was significantly higher than in group $B(p=0.015)$. No significant difference between the numbers of oocytes with $\geq 50 \%$ fragmentation was found between the two study groups ( $p=0.102)$. Finally, the rate of blastocyst formation in group $B$ was markedly lower than in group $A$ $(p<0.001)$ (Table 2).

\section{Discussion}

Based on the proposal that oocytes interact closely with surrounding GCs during maturation, we hypothesized that GCs may play a potential role in the maturation, fertilization, and embryo development rates of human immature oocytes in vitro. Our data demonstrated a considerable improvement in the maturation rate and the likelihood of embryo development, but not in the fertilization rate, in immature oocytes cultured with GCs compared to those cultured without GCs. In order to obtain maximum developmental competence, oocytes must achieve both cytoplasmic and nuclear maturity [16]. Nuclear and cytoplasmic maturation are independent processes. Occasionally, cytoplasmic maturation may successfully be completed without nuclear maturation. GCs not only control cytoplasmic maturation, but also play an important role in nuclear maturation by responding to gonadotropins during folliculogenesis [11]. Communication between oocytes and their surrounding GCs through gap junctions is

Table 1. Clinical data regarding the subjects and the in vitro maturation of oocytes

\begin{tabular}{lccc}
\hline & Group A & Group B & $p$-value \\
\hline No. of cycles & 49 & 47 & - \\
Age $(\mathrm{yr})^{\mathrm{a})}$ & $22.2 \pm 2.0(21.4-22.9)$ & $23.0 \pm 1.9(22.3-23.7)$ & 0.125 \\
Basal FSH (mlU/mL) & $7.41 \pm 1.36(6.86-7.95)$ & $7.88 \pm 0.96(7.49-8.26)$ & 0.156 \\
No. of total oocytes & 97 & 95 & 0.695 \\
No. of mature oocytes (\%) & $27(27.8)$ & $32(33.7)$ & 0.384 \\
No. of immature oocytes (\%) & $70(72.2)$ & $63(66.3)$ & 0.514 \\
No. of mature ooyctes after IVM (\%) & $59(84.3)$ & $41(65.1)$ & 0.011 \\
\hline
\end{tabular}

FSH, follicle-stimulating hormone; IVM, in vitro maturation.

${ }^{a}$ Values are mean $\pm S D$, (range).

Table 2. Comparison of fertilization and embryo development from in vitro matured immature oocytes in the study groups

\begin{tabular}{lccc}
\hline & Group A & Group B & p-value \\
\hline Mature oocytes & 59 & 41 & - \\
Fertilized oocytes & $49(83.1)$ & $33(80.5)$ & 0.702 \\
2PN & $45(91.8)$ & $30(90.9)$ & $3(9.1)$ \\
1PN or 3PN & $4(8.2)$ & $28(93.3)$ & 0.417 \\
Cleavage stage embryos & $44(97.8)$ & $12(42.9,12 / 28)$ & 0.006 \\
Available embryos & $33(75.0,33 / 44)$ & $8(28.6)$ & 0.003 \\
Top quality embryos & $28(63.6)$ & $17(60.7)$ & 0.022 \\
$\geq 6$ cell embryo & $37(84.1)$ & $11(39.3)$ & 0.015 \\
Fragments <10\% embryos & $30(68.2)$ & $10(35.7)$ & 0.102 \\
Fragments $>50 \%$ embryos & $8(18.2)$ & $7(25.0)$ & $<0.001$ \\
Blastocysts & $31(70.5)$ & & \\
\hline
\end{tabular}

Values are presented as number (\%).

$\mathrm{PN}$, pronuclei. 
vital for the development of competent oocytes [17]. Regulatory molecules, glucose metabolites, nucleotides, and amino acids are known to be transferred through the oocyte-GC gap junctions of growing oocytes $[18,19]$. In the present study, GV-stage oocytes cultured with GCs showed improved nuclear maturation rates. These findings are in agreement with those of Goud et al. [20], who reported that the nuclear maturation and cleavage rates in granulosa-intact oocytes were higher than in granulosa-denuded human GV-stage oocytes. Hwang et al. [21] also demonstrated that the maturation rate of oocytes with GCs was markedly higher than that of denuded oocytes, whereas Johnson et al. [13] reported that human M I-stage oocytes cultured with GCs did not show a significantly different rate of oocyte maturation. However, GV-stage oocytes cultured with GCs matured at a greater rate than those cultured without GCs [13].

IVM followed by fertilization opens the novel possibility of fully using the immature oocytes retrieved from controlled ovarian stimulation cycles. Several studies have been and are currently being conducted to characterize the optimal use of immature oocytes; however, the influence of GCs on the developmental potential of human immature oocytes has not been examined in detail. In this regard, different approaches to IVM have obtained results similar to our findings. In accordance with the results of our study, Shu et al. [22] showed that immature oocytes from stimulated cycles can be fertilized normally and used to increase the number of embryos available for transfer. In another study, Strassburger et al. [23] showed significantly higher fertilization rates among embryos from rescued IVM-MII oocytes and more blastomeres per embryo compared with those from arrested MI oocytes. With the goal of examining the influence of GCs on the developmental potential of human immature oocytes, the present study has shown that culturing immature human oocytes with GCs prior to ICSI improves the maturation rate and the likelihood of embryo development. The exact mechanisms responsible for the effect of GCs on early embryonic development remain undefined.

In accordance with previous studies [16,22], we demonstrated that immature human oocytes cultured with and without GCs had similar fertilization rates. However, in contrast to our data, some studies have shown lower rates of fertilization in GC-free immature oocytes in IVM [23-25]. These discrepancies are likely due to differences in the stimulation protocol, the laboratory protocols, the culture medium, the duration of in vitro culture, the timing of oocyte denudation, and the time interval in IVM between sperm administration and the first polar body extrusion.

According to the findings of our study, culturing immature human oocytes without GCs results in an increased fragmentation rate and delayed embryo development, which compromise developmental competence and the quality of the embryo. The presence of GCs for 24 hours of culture led to a marked improvement in the quality of available embryos after oocyte maturation was observed in vitro before ICSI. Moreover, we showed that the blastocyst formation rate from the immature oocytes cultured with GCs was higher than that from the oocytes cultured without GCs. So far, the exact mechanism behind the effect of GCs on early embryonic development is not entirely clear. After IVM, most oocytes achieved nuclear maturation, but were still cytoplasmically immature. In this regard, the asynchrony between nuclear and cytoplasmic maturation in oocytes could compromise the developmental potential of the embryo [26]. It has been previously suggested that co-culture with $\mathrm{GCs}$, starting at different stages of IVF, has no effect on cleavage development and fertilization, but markedly improves the rates of embryo development to the blastocyst or morula stages [27]. Furthermore, it has been demonstrated that granulosa-intact primate oocytes are vital for successful IVM. It has been shown that GCs can produce epidermal growth factor-like substances, including epiregulin, betacellulin, and amphiregulin, which play a fundamental role during the IVM of primate oocytes [28]. Altogether, we think that it is probable that GCs improve the quality of embryos via their effects on the cytoplasmic maturation of in vitro matured oocytes.

Culture conditions and culture media components can modulate the meiotic regulation of oocytes [29]. Media for the IVM of human oocytes have been supplemented with $\mathrm{hCG}$, pregnant mare serum gonadotropin, $\mathrm{FSH}$, estradiol, and human menopausal gonadotropin [30]. However, these supplements may not influence GC-free oocytes to the desired extent, because the receptors of the above molecules are on the GCs [31]. For this reason, $10 \%$ synthetic serum substitute supplemented with HTF medium, without extra supplements, was used in this study to culture immature oocytes. HTF medium without supplemental hormones was similarly used for culturing immature human oocytes with GCs in a previous study [13].

The poor cytoplasmic maturation of oocytes may be the reason for defects in embryonic development [32]. The cytoplasmic maturation of oocytes involves progressing through complicated stages that prepare the cytoplasm of the oocytes for fertilization and activation [33]. During these stages, proteins, imprinted genes, and RNA molecules accumulate in the cytoplasm of oocytes to modulate meiosis and oocyte development [34]. During the ICSI procedure, the abnormal dispersion of chromosomes in the oocyte and premature condensation of the sperm chromatin can occur due to cytoskeletal anomalies and cytoplasmic immaturity in oocytes, influencing the likelihood of embryo formation [35]. Most 3-PN zygotes result from oocytes that undergo premature irregular cytokinesis or retention of the second polar body [36]. It has been previously demonstrated that IVM medium supplementation with GSH-OEt (Sigma) improved the rates of maturation and normal fertilization in oocytes compared to non-supplemented medium [37]. With this in mind, we suggest that culture con- 
ditions may play a crucial role for the IVM of human oocytes, in addition to genetic factors in the immature oocytes.

According to the findings of the present study, the nuclear maturation rates were not significantly different between the oocytes co-cultured with GCs and those denuded of GCs. We suggest that this effect may be mediated by improved cytoplasmic maturation [38]. It has been shown that GCs are critical for complete cytoplasmic maturation in oocytes in rats, pigs, sheep, mice, and cattle $[26,39,40]$. After IVM, most oocytes are mature in the nucleus but immature in the ooplasm. This asynchrony of the maturation process may compromise the developmental potential of the oocyte [26]. It has been shown that a coculture of GCs starting at various IVF stages had no effect on cleavage development and fertilization, but significantly improved the rates of embryo development to the morula and blastocyst stages [41]. Moreover, in the rhesus monkey, successful IVM has only been achieved with granulosa-enclosed oocytes, indicating that the presence of intact GCs is crucial for IVM [28].

A strength of this study was its homogenous population due to the application of strict inclusion and exclusion criteria. The presence of insignificant differences in fertilization rates between the two study groups may be attributed to the small number of retrieved immature oocytes, resulting in insufficient power, which is a limitation of this study. In the present study, only immature GV-stage oocytes were stripped for IVM. The exact reason for this is not entirely clear, but the flexible GnRH antagonist protocol that was used in this study involved this methodological approach. In the future, more research should focus on this issue.

In conclusion, the findings of this study suggest that culturing immature human oocytes with GCs prior to ICSI improves the maturation rates and the likelihood of embryo development. According to our findings, delayed or incomplete oocyte cytoplasmic maturation may play a crucial role in the low developmental potential of GC-free oocytes. It is suggested that future studies focus on the use of specialized media for the IVM of immature oocytes.

\section{Conflict of interest}

No potential conflict of interest relevant to this article was reported.

\section{References}

1. Chang EM, Song HS, Lee DR, Lee WS, Yoon TK. In vitro maturation of human oocytes: its role in infertility treatment and new possibilities. Clin Exp Reprod Med 2014;41:41-6.

2. Pincus $G$, Enzmann EV. The comparative behavior of mammalian eggs in vivo and in vitro: I. the activation of ovarian eggs. J Exp Med 1935;62:665-75.
3. Fukuda Y, Ichikawa M, Naito K, Toyoda Y. Birth of normal calves resulting from bovine oocytes matured, fertilized, and cultured with cumulus cells in vitro up to the blastocyst stage. Biol Reprod 1990;42:114-9.

4. Cha KY, Koo JJ, Ko JJ, Choi DH, Han SY, Yoon TK. Pregnancy after in vitro fertilization of human follicular oocytes collected from nonstimulated cycles, their culture in vitro and their transfer in a donor oocyte program. Fertil Steril 1991;55:109-13.

5. Trounson A, Wood C, Kausche A. In vitro maturation and the fertilization and developmental competence of oocytes recovered from untreated polycystic ovarian patients. Fertil Steril 1994;62: 353-62.

6. Guigon CJ, Cohen-Tannoudji M. Reconsidering the roles of female germ cells in ovarian development and folliculogenesis. Biol Aujourdhui 2011;205:223-33.

7. Sugiura K, Pendola FL, Eppig JJ. Oocyte control of metabolic cooperativity between oocytes and companion granulosa cells: energy metabolism. Dev Biol 2005;279:20-30.

8. Wigglesworth K, Lee KB, O'Brien MJ, Peng J, Matzuk MM, Eppig JJ. Bidirectional communication between oocytes and ovarian follicular somatic cells is required for meiotic arrest of mammalian oocytes. Proc Natl Acad Sci U S A 2013;110:E3723-9.

9. Su YQ, Sugiura K, Wigglesworth K, O'Brien MJ, Affourtit JP, Pangas $S A$, et al. Oocyte regulation of metabolic cooperativity between mouse cumulus cells and oocytes: BMP15 and GDF9 control cholesterol biosynthesis in cumulus cells. Development 2008; 135:111-21.

10. Tatemoto H, Sakurai N, Muto N. Protection of porcine oocytes against apoptotic cell death caused by oxidative stress during in vitro maturation: role of cumulus cells. Biol Reprod 2000;63: 805-10.

11. Russell JB. Immature oocyte retrieval combined with in-vitro oocyte maturation. Hum Reprod 1998;13 Suppl 3:63-70.

12. Lainas TG, Sfontouris IA, Zorzovilis IZ, Petsas GK, Lainas GT, Alexopoulou E, et al. Flexible $\mathrm{GnRH}$ antagonist protocol versus $\mathrm{GnRH}$ agonist long protocol in patients with polycystic ovary syndrome treated for IVF: a prospective randomised controlled trial (RCT). Hum Reprod 2010;25:683-9.

13. Johnson JE, Higdon HL 3rd, Boone WR. Effect of human granulosa cell co-culture using standard culture media on the maturation and fertilization potential of immature human oocytes. Fertil Steril 2008;90:1674-9.

14. Van Steirteghem AC, Liu J, Joris H, Nagy Z, Janssenswillen C, Tournaye $\mathrm{H}$, et al. Higher success rate by intracytoplasmic sperm injection than by subzonal insemination: report of a second series of 300 consecutive treatment cycles. Hum Reprod 1993;8:1055-60.

15. Puissant F, Van Rysselberge M, Barlow P, Deweze J, Leroy F. Em- 
bryo scoring as a prognostic tool in IVF treatment. Hum Reprod 1987;2:705-8.

16. Reichman DE, Politch J, Ginsburg ES, Racowsky C. Extended in vitro maturation of immature oocytes from stimulated cycles: an analysis of fertilization potential, embryo development, and reproductive outcomes. J Assist Reprod Genet 2010;27:347-56.

17. Mori T, Amano T, Shimizu H. Roles of gap junctional communication of cumulus cells in cytoplasmic maturation of porcine oocytes cultured in vitro. Biol Reprod 2000;62:913-9.

18. Su YQ, Wu X, O'Brien MJ, Pendola FL, Denegre JN, Matzuk MM, et al. Synergistic roles of BMP15 and GDF9 in the development and function of the oocyte-cumulus cell complex in mice: genetic evidence for an oocyte-granulosa cell regulatory loop. Dev Biol 2004;276:64-73.

19. Araujo VR, Gastal MO, Figueiredo JR, Gastal EL. In vitro culture of bovine preantral follicles: a review. Reprod Biol Endocrinol 2014; 12:78.

20. Goud PT, Goud AP, Qian C, Laverge H, Van der Elst J, De Sutter P, et al. In-vitro maturation of human germinal vesicle stage oocytes: role of cumulus cells and epidermal growth factor in the culture medium. Hum Reprod 1998;13:1638-44.

21. Hwang JL, Lin YH, Tsai YL. In vitro maturation and fertilization of immature oocytes: a comparative study of fertilization techniques. J Assist Reprod Genet 2000;17:39-43.

22. Shu Y, Gebhardt J, Watt J, Lyon J, Dasig D, Behr B. Fertilization, embryo development, and clinical outcome of immature oocytes from stimulated intracytoplasmic sperm injection cycles. Fertil Steril 2007;87:1022-7.

23. Strassburger D, Friedler S, Raziel A, Kasterstein E, Schachter M, Ron-El R. The outcome of ICSI of immature MI oocytes and rescued in vitro matured MII oocytes. Hum Reprod 2004;19:1587-90.

24. Choi BH, Bang JI, Jin Jl, Kim SS, Jo HT, Deb GK, et al. Coculturing cumulus oocyte complexes with denuded oocytes alters zona pellucida ultrastructure in in vitro matured bovine oocytes. Theriogenology 2013;80:1117-23.

25. Dey SR, Deb GK, Ha AN, Lee Jl, Bang Jl, Lee KL, et al. Coculturing denuded oocytes during the in vitro maturation of bovine cumulus oocyte complexes exerts a synergistic effect on embryo development. Theriogenology 2012;77:1064-77.

26. Tao Y, Cao C, Zhang M, Fang F, Liu Y, Zhang Y, et al. Effects of cumulus cells on rabbit oocyte in vitro maturation. J Anim Physiol Anim Nutr (Berl) 2008;92:438-47.

27. Wongsrikeao P, Kaneshige Y, Ooki R, Taniguchi M, Agung B, Nii M, et al. Effect of the removal of cumulus cells on the nuclear maturation, fertilization and development of porcine oocytes. Reprod Domest Anim 2005;40:166-70.
28. Nyholt de Prada JK, Lee YS, Latham KE, Chaffin CL, VandeVoort CA. Role for cumulus cell-produced EGF-like ligands during primate oocyte maturation in vitro. Am J Physiol Endocrinol Metab 2009;296:E1049-58.

29. Downs SM, Mastropolo AM. Culture conditions affect meiotic regulation in cumulus cell-enclosed mouse oocytes. Mol Reprod Dev 1997;46:551-66.

30. Souza-Fabjan JM, Corbin E, Locatelli Y, Duffard N, Perreau C, Freitas VJ, et al. 306 effect of different in vitro maturation media on developmental potential of goat oocytes already found denuded at collection. Reprod Fertil Dev 2014;27:242.

31. Cheng KW, Leung PC. The expression, regulation and signal transduction pathways of the mammalian gonadotropin-releasing hormone receptor. Can J Physiol Pharmacol 2000;78:1029-52.

32. Salamone DF, Damiani P, Fissore RA, Robl JM, Duby RT. Biochemical and developmental evidence that ooplasmic maturation of prepubertal bovine oocytes is compromised. Biol Reprod 2001; 64:1761-8.

33. Cha KY, Chian RC. Maturation in vitro of immature human oocytes for clinical use. Hum Reprod Update 1998;4:103-20.

34. Ruddock NT, Wilson KJ, Cooney MA, Korfiatis NA, Tecirlioglu RT, French AJ. Analysis of imprinted messenger RNA expression during bovine preimplantation development. Biol Reprod 2004;70: 1131-5.

35. Zhivkova R, Delimitreva S, Vatev I. Role of oocyte cytoplasmic factors in human IVF failure. Akush Ginekol (Sofiia) 2010;49:26-32.

36. Rosenbusch BE, Schneider M. Separation of a pronucleus by premature cytokinesis: a mechanism for immediate diploidization of tripronuclear oocytes? Fertil Steril 2009;92:394.e5-8.

37. Curnow EC, Ryan JP, Saunders DM, Hayes ES. In vitro developmental potential of macaque oocytes, derived from unstimulated ovaries, following maturation in the presence of glutathione ethyl ester. Hum Reprod 2010;25:2465-74.

38. Watson AJ. Oocyte cytoplasmic maturation: a key mediator of oocyte and embryo developmental competence. J Anim Sci 2007; 85(13 Suppl):E1-3.

39. Godard NM, Pukazhenthi BS, Wildt DE, Comizzoli P. Paracrine factors from cumulus-enclosed oocytes ensure the successful maturation and fertilization in vitro of denuded oocytes in the cat model. Fertil Steril 2009;91(5 Suppl):2051-60.

40. Tanghe S, Van Soom A, Mehrzad J, Maes D, Duchateau L, de Kruif A. Cumulus contributions during bovine fertilization in vitro. Theriogenology 2003;60:135-49.

41. Zhang L, Jiang S, Wozniak PJ, Yang X, Godke RA. Cumulus cell function during bovine oocyte maturation, fertilization, and embryo development in vitro. Mol Reprod Dev 1995;40:338-44. 